\title{
Knowledge on Risk Factors of Coronary Heart Disease among Middle Aged Administrative Staffs of Kathmandu
}

\section{Soni Shrestha ${ }^{1} \&$ Deepa Gautam ${ }^{2}$}

${ }^{1}$ Associate Professor, Man Mohan Memorial Institute of Health Science, Kathmandu

${ }^{2}$ Nursing Instructor, Bharatpur Nursing College

\section{Corresponding Author}

Soni Shrestha

Email-sonishrestha2003@yahoo.com

\begin{abstract}
Background: Progressive urbanization and adoption of the "western" lifestyle contributes to the rising burden of cardiovascular diseases in the developing world. Coronary Heart Disease (CHD) is no longer confined by geographical area or socioeconomic boundary. Knowledge is an important pre-requisite for implementing both primary and secondary preventive strategies of CHD and for behavioral change.

Objective: This study attempts to quantify level of knowledge on risk factors of Coronary Heart Disease among middle aged administrative staffs of Tribhuvan University, Kritipur Kathmandu.

Methodology: Descriptive Cross Sectional study was conducted in different departments of Tribhuvan University, Kathmandu from December 22, 2014 to January 2, 2015.A total of 130 middle aged administrative staffs (40-60 years) have participated in the study. Structured questionnaires and interview schedule were used to collect data and descriptive statistics was used for data analysis.

Results: Among total respondents, $56.2 \%$ had mean knowledge score equal to or more than 13.91. Only less than half (47.7\%) knew age as non - modifiable risk factor of CHD followed by sex $(30.8 \%)$ and family history (30\%). Regarding modifiable risk factors, most participant response was fatty food consumption (80.8\%) followed by tobacco use (80\%), excessive alcohol consumption (78.5\%), hypertension(76.2\%), Stress(74.6\%), lackofexercise/inactivelifestyle (73.8\%), Obesity (70.8\%), excessive salt consumption in diet (60.8\%) and diabetes mellitus(46.9\%). In regards to the symptom of CHD, most cited response was chest pain/discomfort(55\%). The risk factors prevalent among the study participants included overweight (44.6\%) and alcohol consumption (30.8\%)
\end{abstract}

Conclusion: The findings concluded that significant percentage (43.8\%) of respondent's mean knowledge score was less than 13.91. Thus, understanding of the behavioral determinants of heart disease knowledge and risk factors is necessary in designing effective prevention strategies. 
KEY WORDS: Coronary Heart Disease, Knowledge, Middle Aged Adults, Risk Factors

\section{INTRODUCTION}

Coronary heart disease (CHD) is the most common type of heart disease and cause of heart attack. Incidence of CHD occurs in men between 35 to 45 years age. After the age of 65 the incidence of men and women equalizes, although there is evidence suggesting that more women are being seen with CHD earlier because of increased stress, smoking and menopause(Basavantthapa, 2012).Cardiovascular diseases(CVDs) are the number one cause of death globally with more deaths resulting annually from CVDs than from any other cause. It has been estimated that about 17.3 million people died from CVD in 2008, representing $30 \%$ of all global deaths. Of these deaths, an estimated 7.3 million were due to coronary heart disease and 6.2 million were due to stroke. Low and middle income countries are disproportionally affected resulting in over $80 \%$ of deaths in these countries almost equally in men and women (World Health Organization [WHO], 2010). The risk of coronary heart disease increases as age increases. Middle aged adults are mostly affected by CHD .For men, the risk starts to climb at about age 45 , and by age 55 , the risk becomes double. It continues to increase until, by age 85 . For women, the risk of coronary heart disease also climbs with age, but the trend begins about 10 years later than in men and especially with the onset of menopause. Coronary heart disease has become one of the major public health problems in Nepal. According to the world health data published in 2011, coronary heart disease deaths in Nepal reached 21,152 or $14.24 \%$ of total deaths and age standardized death rate is 152.62 per 100,000 population with Nepal ranking in $47^{\text {th }}$ position in the world (WHO, 2010)

Therefore, it has become more essential to prevent the development of CHD and reduce its burden among middle aged adults through primary and secondary prevention of coronary heart disease (Society for Cardiovascular Angiography and Interventions [SCAI], 2014). Studies conducted in Nepal also revealed low knowledge of Coronary heart disease among the general public. A study conducted to assess knowledge of modifiable risk factors of coronary atherosclerotic heart disease in Kathmandu showed that only $22 \%$ of study population had good level of knowledge regarding the risk factors (Khadka,2012).Another similar study done in Kathmandu revealed that $67.8 \%$ of participants had adequate knowledge regarding heart disease among 405 participants (Pandey \& Khadka, 2012). Ageing populations and rapidly changing lifestyles, the burden of CHD is increasing in almost all developing countries including Nepal. Apart from the hospital-based data that show a steady rise in the number of patients suffering from CHD, community-based studies in Nepal have also indicated a high prevalence of the disease and its risk factors in the general population (Vaidya, 2008). 
Although coronary heart disease incidences is increasing in Nepal, it can be highly preventable as it is caused by many modifiable risk factors like smoking cigarette and tobacco use ,alcohol consumption, obesity, physical inactivity, unhealthy diet, stress, high blood pressure etc. Therefore, it is important that people know about the measures to prevent coronary heart disease by adopting healthy behaviors like being physically active, consuming healthy diet, abstaining from smoking and alcohol and keeping blood pressure under control. For prevention and reduction of the burden of coronary heart disease it is essential that people have knowledge of risks of Coronary heart disease so that they assume themselves at risk and adopt measures to prevent the disease.

Thus, the findings of this study will provide baseline information about knowledge of coronary heart disease and its risk factors among the middle aged adults and identify the gaps in knowledge level as the modification in behavior will not take place without adequate awareness of the health problem.

The findings of this study might be helpful for researchers, students in doing further study regarding knowledge of risk factors of CHD. Furthermore, the findings of this study might help to develop educational programs for the risk groups aiming to generate awareness regarding coronary heart disease and ways of preventing it.

\section{METHODS}

Descriptive Cross-sectional study was conducted from December 22, 2014 to January 2, 2015. .A total of 130 middle aged administrative staffs (40-60 years) of Tribhuvan University Central Office have participated in the study. 130 staffs were selected randomly for the study using simple random sampling technique. Structured questionnaires were developed for collecting the data. Questionnaires were developed through extensive literature review of related topics. Questionnaires consisted of four parts. Part I consisted questions for assessing sociodemographic characteristics and co-morbidities of the respondents. Part II consisted questions related to coronary heart disease risk behaviors. Part III consisted questions related to knowledge of CHD, risk factors and prevention of CHD. Informed consent was taken from all the study participants. Part IV consisted of physical measurements-height and weight Formal approval was taken from Institutional Review Committee, IRC, MMIHS. Formal permission was obtained from the authority of study area. Verbal consent was taken from each respondent. Privacy, confidentiality and anonymity of the respondents were maintained. The data analysed in the statistical package for social science software (SPSS) 17 versions and analyzed using descriptive statistics. The results were reported as frequency and percentages. Obtained findings were interpreted in tabular form.

\section{RESULTS}


One Hundred thirty respondents were interviewed. Among 130, $38.5 \%$ of the respondents belonged to age group 40-46 years, $34.6 \%$ of the respondents were of age group 47-53 years and $26.9 \%$ were of age group 54-60 years. The mean age of the respondents was 49.8 years. More than half $63.1 \%$ of the respondents were male and remaining $36.9 \%$ were female. Majority of the respondents $83.1 \%$ were Hindu, $14.6 \%$ were Buddhist, $1.5 \%$ were Christian and $0.8 \%$ was Kirat. Among 130 respondents, $17.7 \%$ of the respondents had family history of heart disease. $16.2 \%$ of the total respondents had self reported hypertension. Likewise, $9.2 \%$ of the respondents had self reported diabetes .According to BMI classification, equal proportion of respondents i.e. $44.6 \%$ were of normal and overweight and $10.8 \%$ of the respondents were obese. Table-1

Family history of heart disease, self reported presence of hypertension, diabetes and BMI classification

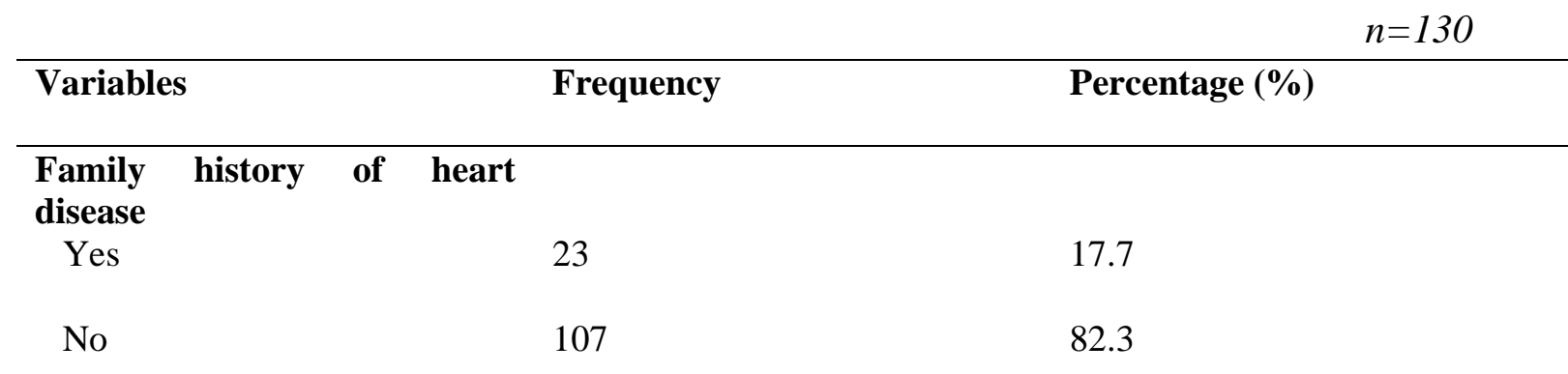

\section{Hypertension}

Yes

No

Diabetes

Yes

No

\section{BMI classification}

Normal weight 58 44.6

Overweight 58

14
16.2

83.8
9.2

90.8
Obese 118 .

- Normal weight(BMI: $18.5-24.9 \mathrm{~kg} / \mathrm{m}^{2}$ )

- Overweight (BMI: $25-29.9 \mathrm{~kg} / \mathrm{m}^{2}$ ) 
- $\operatorname{Obese}\left(B M I>30 \mathrm{~kg} / \mathrm{m}^{2}\right)$

Regarding the Coronary Heart Diseases risk factors among 130 respondents, majority of the respondents $70.8 \%$ were lifetime abstainers, $17.7 \%$ were current smokers and $11.5 \%$ were ex smokers. Among current smokers, all $17.7 \%$ smoked cigarettes. Majority $39.1 \%$ of the respondents smoked for 20-29years, 26.1\% smoked for 10-19 years, $21.7 \%$ of the respondents smoked for 30-39 years and 13\% of the respondents smoked for 40-49 years. Among 23 smokers, majority $39.1 \%$ of the respondents consumed 5-9 cigarettes in a day followed by less than 5 cigarettes and 10-14 cigarettes by $30.4 \%, 17.4 \%$ and $13 \%$ consumed 15-24 cigarettes a day. The prevalence of smokeless tobacco use was $10 \%$ of the total respondents with all involved in chewing tobacco (Pan Masala, khaini).

Table-2. Tobacco use (smoking habit)

\begin{tabular}{lll}
\multicolumn{1}{c}{$n=130$} & Frequency & Percentage (\%) \\
\hline Description & 92 & 70.8 \\
$\begin{array}{l}\text { Tobacco use(smoking habit) } \\
\text { Never used }\end{array}$ & 15 & 11.5 \\
Ex user & 23 & 17.7 \\
Current user & & \\
\hline
\end{tabular}

Type, duration and quantity of smoking done by current smokers

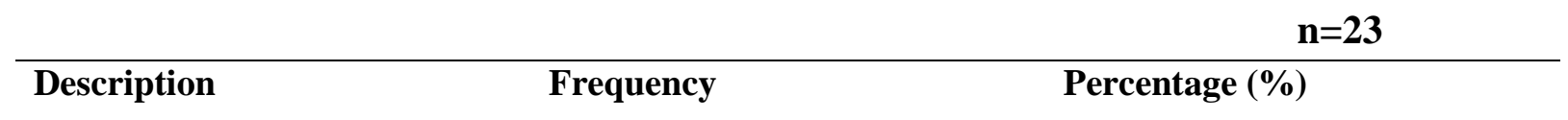

\section{Form of smoking used}

Cigarette

\section{Duration of cigarette consumption} 10-19 years

20-29 years

30-39 years

$40-49$ years

Quantity of cigarettes consumed in a day

Less than 5 cigarettes
26.1

3 
5-9 cigarettes

10-14 cigarettes

15-24 cigarettes
9

4

3
39.1

17.4

13

Thirty one percent $(n=40)$ were found to be current alcohol drinkers while $61.5 \%$ were lifetime abstainers and $7.7 \%$ were past drinkers. Among 40 alcohol drinkers, majority $40 \%$ consumed alcohol for 10-19 years, 32.5\% consumed alcohol for 20-29 years, other $15 \%$ consumed alcohol for 40-49 years and 12.5\% consumed alcohol for 30-39 years. Among 40 alcohol drinkers, majority $30 \%$ of alcohol drinkers consumed alcohol1-4 days a week, 20\% consumed alcohol 5-6 days a week, $17.5 \%$ were daily consumers and less than once a month respectively and $15 \%$ of the alcohol drinkers consumed alcohol 1-3 days per month.

Among 130 respondents, majority $73.8 \%$ of the respondents were involved in some form of exercise. In regards to the type of exercise done by the respondents, majority $80.2 \%$ were involved in walking. Household activity, jogging and running was done by $19.8 \%, 16.7 \%$ and $11.5 \%$ respectively. Regarding at least 30 minutes of exercise per week done by the respondents, more than half of the respondents $57.3 \%$ did 3-4 times exercise per week, $24 \%$ did 0-2 times exercise per week whereas $18.8 \%$ of the respondents did 5 or more times exercise per week.

Among 130 respondents, majority $80.8 \%$ of the respondents eats non- vegetarian diet and only $19.2 \%$ of the respondents eat vegetarian diet. Likewise, in regards to the amount of fruits and vegetables consumed a day, most of the respondents $75.4 \%$ had 1 serving of fruits and vegetables a day and other $24.6 \%$ had 2 servings of fruits and vegetables in a day. Comparing this to the minimum recommended intake, all of the respondents did not consume adequate amount of fruits and vegetables per day.

Among 130 respondents, $80 \%$ of the respondents knew that tobacco consumption is a risk factor of developing CHD .Likewise, $78.5 \%$ of the respondents knew excessive alcohol consumption as a risk factor of CHD, $17.7 \%$ of the respondents didn't know that excessive alcohol consumption is a risk factors. Among total respondents, $73.8 \%$ of the respondents knew lack of exercise/inactive lifestyle as a risk factor of CHD. Regarding obesity, 70.8\% of the respondents knew obesity as a risk factor of CHD. $80.8 \%$ of the respondents knew that fatty food consumption is a risk factor of developing CHD whereas $16.2 \%$ didn't know that fatty food consumption is a risk factor of developing CHD.Among total respondents, $60.8 \%$ of the respondents knew excessive salt consumption in diet is a risk factor of CHD, 30\% of the respondents didn't know that excessive salt consumption is a risk factor and $9.2 \%$ said that excessive salt consumption is not a risk factor of CHD. 
Similarly, $76.2 \%$ of the respondents knew hypertension is a risk factor of CHD. Regarding diabetes, $46.9 \%$ of the respondents knew diabetes is a risk factor of CHD. $74.6 \%$ of the respondents knew stress is a risk factor of $\mathrm{CHD}, 47.7 \%$ of the respondents knew increasing age is a risk factor of developing CHD, 28.5\% didn't know increasing age is a risk factor of developing CHD Likewise, majority of the respondents $37.7 \%$ didn't know men are at greater risk of developing CHD, $31.5 \%$ of the respondents mentioned that men are not at increasing risk of developing CHD while only $30.8 \%$ of the respondents knew that men are at increasing risk of developing CHD.

Among total respondents, $37.7 \%$ of the respondents didn't know that family history of CHD is a risk factor of developing CHD, among various risk factors, most mentioned risk factor by the respondents was fatty food consumption while least mentioned risk factor was family history of CHD.

Table-3. Knowledge regarding CHD, risk factors and its prevention $\mathbf{n}=\mathbf{1 3 0}$

\begin{tabular}{lll}
\hline Description & Frequency & Percentage (\%)
\end{tabular}

\section{Cause of CHD}

Age only

Obstruction of arteries of the 90

69.2

heart

Don't know

CHD is a type of disease which

Lasts for a short time

Comes and goes with season

1

Will remain for a long period

Don't know

*Symptom of CHD

Chest pain/discomfort

Shortness of breath

Feeling weak, light headed or 33 faint

Don't know

\section{5}

38

24.8

12.4 


\begin{tabular}{|c|c|c|c|c|c|c|}
\hline \multirow[t]{2}{*}{ Variables } & \multicolumn{2}{|l|}{ Yes } & \multicolumn{2}{|l|}{ No } & \multicolumn{2}{|l|}{ Don't know } \\
\hline & Frequency & Percentage & Frequency & Percentage & Frequency & Percentage \\
\hline $\begin{array}{l}\text { Tobacco } \\
\text { consumption(smoke } \\
\text { and } \quad \text { smokeless } \\
\text { tobacco) }\end{array}$ & 104 & 80 & 8 & 6.2 & 18 & 13.8 \\
\hline $\begin{array}{l}\text { Excessive alcohol } \\
\text { consumption }\end{array}$ & 102 & 78.5 & 5 & 3.8 & 23 & 17.7 \\
\hline $\begin{array}{l}\text { Lack } \\
\text { exercise/inactive } \\
\text { lifestyle }\end{array}$ & 96 & 73.8 & 7 & 5.4 & 27 & 20.8 \\
\hline Obesity & 92 & 70.8 & 14 & 10.8 & 24 & 18.5 \\
\hline $\begin{array}{ll}\text { Fatty } & \text { food } \\
\text { consumption } & \end{array}$ & 105 & 80.8 & 4 & 3.1 & 21 & 16.2 \\
\hline $\begin{array}{l}\text { Excessive salt } \\
\text { consumption in diet }\end{array}$ & 79 & 60.8 & 12 & 9.2 & 39 & 30 \\
\hline Hypertension & 99 & 76.2 & 9 & 6.9 & 22 & 16.9 \\
\hline Diabetes & 61 & 46.9 & 23 & 17.7 & 46 & 35.4 \\
\hline Stress & 97 & 74.6 & 8 & 6.2 & 25 & 19.2 \\
\hline Increasing age & 62 & 47.7 & 31 & 23.8 & 37 & 28.5 \\
\hline Sex & 40 & 30.8 & 41 & 31.5 & 49 & 37.7 \\
\hline $\begin{array}{l}\text { Family history of } \\
\text { CHD }\end{array}$ & 39 & 30 & 42 & 32.3 & 49 & 37.7 \\
\hline
\end{tabular}

Majority of the respondents $90 \%$ knew that CHD is preventable .Among different preventive measures majority of the respondents $70.9 \%$ mentioned eating plenty of fruits and vegetables, $69.2 \%$ mentioned decreasing consumption of fatty foods and $66.7 \%$ mentioned daily physical exercise and abstaining from alcohol and tobacco consumption as a preventive measure of CHD respectively. Likewise, 59\%identified keeping blood pressure under control as preventive measure, 51.3\%identified stress reduction and $47 \%$ mentioned avoiding consumption of excessive salt in diet.

The knowledge score of 130 respondents revealed that mean knowledge score was 13.91. More than half $56.2 \%$ of the respondent's mean knowledge score was more than or equal to 13.91 whereas $43.8 \%$ of the respondent's mean knowledge score was less than 13.91 .

Knowledge score of the respondents

$$
\mathbf{n}=\mathbf{1 3 0}
$$




\begin{tabular}{|c|c|c|}
\hline Mean knowledge score & Frequency & Percentage (\%) \\
\hline$<13.91$ & 57 & 43.8 \\
\hline$\geq 13.91$ & 73 & 56.2 \\
\hline Total & 130 & 100 \\
\hline
\end{tabular}

\section{DISCUSSION}

Socio demographic findings of this study revealed that $38.5 \%$ of the respondents were of age group 40-46 years and mean age of the respondents was 49.78 years which is similar to the study conducted in Southern Nevada which showed majority of the study subjects of age group 42-49 years with the mean age of 50.8 years (Angosta \& Speck, 2014).

Among 130 respondents, $17.7 \%$ had family history of heart disease which showed consistency with the study conducted in South India by (Varghese, 2014) where 24\% of study participants had family history of heart disease.

Regarding the presence of hypertension and diabetes, $16.2 \%$ and $9.2 \%$ of the total respondents had hypertension and diabetes respectively which is congruous with the study done by (Saeed et al; 2009) which showed $8.9 \%$ hypertension, $6.6 \%$ diabetes. Similarly, another study done in Pakistan by (Jafary et al; 2005) showed that $14.9 \%$ had hypertension and $5.9 \%$ had diabetes respectively.

In a study conducted in Kuwait, $35.2 \%, 38.1 \%$ and $24.3 \%$ of the study subjects were of normal weight, overweight and obese(Awad \& Al-Nafisi,2014) that showed consistency with this study where $44.6 \%$ of respondents were of normal weight and overweight respectively and $10.8 \%$ were obese.

This study exhibited that $17.7 \%$ were current smokers, $10 \%$ were current smokeless tobacco users and 30.8\% were current alcohol drinkers among total study subjects but this finding is contradictory to the study conducted in Pakistan, Kathmandu and Kazakhstan (Jafary et al;2005;Khadka,2012;Kulkayeva et al;2012).

In a study conducted in Karachi, Pakistan, it was reported that only $4.3 \%$ of the study subjects were involved in exercise (Khan et al; 2006). Similarly, another study by (Khadka,2012) in Kathmandu revealed that $17.35 \%$ of the total study participants were involved in regular exercise. These results are in contrast with this study which revealed that around two third (73.8 $\%$ ) of the study participants were involved in exercise. Likewise, 3-4 times of exercise per week 
was performed by $42.3 \%$ of the respondents which showed variation to the study conducted in Kuwait where only $13.7 \%$ were involved in 3-4 times exercise per week(Awad \& AlNafisi,2014).

This study revealed that all of the study participant's fruits and vegetables consumption was less than the minimum recommended five daily servings per day, which was consistent with result of national study, NCDs risk factor survey 2013(Aryal et al;2013) which reported that majority 98.9 $\%$ did not consume an adequate amount of fruits and vegetables on a day.

This study exhibited that most of the respondents were able to recognize fatty food consumption, tobacco consumption, lack of exercise and obesity as the risk factors of CHD which is consistent with the study done in Karachi, Pakistan where majority of the respondents knew smoking and fatty food consumption as risk factors(Khan et al;2006). However, the results regarding lack of exercise and obesity is not congruous with the above study.

Among total respondents, (78.5\%) identified excessive alcohol consumption, excessive salt consumption in diet $(60.8 \%)$, hypertension $(76.2 \%)$, diabetes $(46.9 \%)$, stress $(74.6 \%)$, increasing age $(47.7 \%)$ sex $(30.8 \%)$, and family history of heart disease $(30 \%)$ as the risk factors of CHD which is contradictory to the study conducted in Kazakhstan and Pakistan where alcohol drinking recognized by $64.8 \%$, excess dietary salt recognized by only $3.3 \%$,stress by $43.4 \%$ and diabetes by only $1.9 \%$ of the study participants (Kulkayeva \& Jafary; 2012, 2006) However, this finding is invariable with the study done by (Khadka, 2012) where $85.2 \%$ identified hypertension. Another study conducted by (Pandey \& Khadka, 2012) in Kathmandu showed that age, family history was recognized was $46.9 \%$ and $39.8 \%$ of study participants respectively which is similar with the finding of this study. However, sex was recognized by only $13.8 \%$ of respondents which showed distinction with the finding of this study.

In this study decreasing fatty food consumption, eating fruits and vegetables was most recognized by the respondents as the ways to prevent $\mathrm{CHD}$ which is similar to the study conducted by (Pandey \& Khadka, 2012).

\section{CONCLUSION}

Descriptive cross sectional study was conducted to assess the knowledge on risk factors of CHD among middle aged administrative staffs of Tribhuvan University. Based on the findings of the study, it was concluded that the mean knowledge score was found to be 13.91 among the respondents. Thus, $56.2 \%$ of respondents knowledge score was equal to or more than 13.91 but there was a significant percentage $43.8 \%$ of respondents whose knowledge score was less than 13.91 . 
However, more than half of the respondents were able to identify cause of CHD; most mentioned symptom was chest pain. Nearly two third of the respondents identified fatty food consumption and tobacco consumption as risk factors of CHD. Regarding preventive measures, eating fruits and vegetables and decreasing fatty food consumption were most mentioned preventive measures of CHD. But the respondents were less able to identify non-modifiable risk factors of CHD like increasing age, male gender and family history of CHD as the risk factors.

This survey also found prevalence of different modifiable and biological risk factors among the study participants. Among various modifiable risk factors low fruits and vegetables consumption was found in all of the respondents followed by alcohol consumption, tobacco use. On the other hand, among various biological risk factors overweight was seen in nearly half of the respondents followed by hypertension, obesity, diabetes. Prevalence of CHD risk factors among respondents put them at greater risk of developing CHD. Promotion of awareness programs at the primary health care level with emphasis on changing behavior related risk factors can reduce CHD related morbidity and mortality and help for better quality of life of high risk subjects. Also, early screening programs targeting the middle aged and younger adults are important to detect the risk factors in earliest stages and to take appropriate action.

Thus, the overall study exhibits that though more than half of the middle aged administrative staffs had knowledge regarding coronary heart disease; prevalence of CHD risk factors among them is high. Attention need to be paid in the intensive planning of educational programme for prevention of heart disease as prevention of CHD is the most effective way of combating its epidemic in the resource poor nation like Nepal. This study can provide baseline information for further research in related issues. The results of this study would provide a foundation upon which to build programs and policies. The study area was limited to only one organization and covered only the middle aged administrative staffs. Small sample size was covered so it might be difficult to generalize the findings of the study .A further limitation of the study is the cross sectional nature of data that represented one point in time and therefore, do not reflect any changes in respondent's knowledge about CHD.

Different educational programs should be planned targeting middle aged adults for the prevention of CHD. Different screening programs for the prevalence of CHD related behavioral risk factors can be conducted and thus awareness programs can be promoted at the primary health care level with emphasis on changing behavior related risk factors among high risk subjects .A similar study can be conducted on large scale in different setting i.e. in community setting in both rural and urban areas. Similar studies can be carried out among young adults and adolescents group.

\section{REFERENCES}


Angosta, A. D., \& Speck, K. E. (2014). Assessment of heart disease knowledge and risk factors among first-generation Filipino Americans residing in Southern Nevada: A crosssectional survey. Clinical Nursing Studies, 2(2), p123.

Aryal, K., Neupane, S., Mehata, S., Vaidya, A., Singh, S., Paulin, F., Guthold, R. (2014). Non communicable diseases risk factors: STEPS Survey Nepal 2013. Kathmandu: Nepal Health Research Council.

Awad, A., \& Al-Nafisi, H. (2014). Public knowledge of cardiovascular disease and its risk factors in Kuwait: a cross-sectional survey. BMC Public Health, 14(1), 1131.

Jafary, F. H., Aslam, F., Mahmud, H., Waheed, A., Shakir, M., Afzal, A., . . $\quad$ Haque, I. U. (2005). Cardiovascular health knowledge and behavior in patient attendants at four tertiary care hospitals in Pakistan-a cause for concern. BMC Public Health, 5(1), 124.

Khadka, M. (2012). Knowledge regarding modifiable risk factors of coronary atherosclerotic heart disease in kathmandu municipality. NEPALESE heart journal, 9(1), 37-42. 11

Khan, M. S., Jafary, F. H., Jafar, T. H., Faruqui, A. M., Rasool, S. I., Hatcher, J., \& Chaturvedi, N. (2006). Knowledge of modifiable risk factors of heart disease among patients with acute myocardial infarction in Karachi, Pakistan: a cross sectional study. $B M C$ cardiovascular disorders, 6(1), 18

Kulkayeva, G., Rashid, H., Yoshida, Y., Tulebayev, K., \& Sakamoto, J. (2012).CARDIOVASCULAR DISEASE RISK FACTORS AMONG RURAL KAZAKH POPULATION. Nagoya Journal of Medical science, 74, 51-61. 10

Pandey, R. A., \& Khadka, I. (2012). Knowledge regarding preventive measures of heart disease among the adult population in Kathmandu. Health, 4, 601. 3

Saeed, O., Gupta, V., Dhawan, N., Streja, L., Shin, J. S., Ku, M., . . . Verma, S. (2009). Knowledge of modifiable risk factors of Coronary Atherosclerotic Heart Disease (CASHD) among a sample in India. BMC international health and human rights, 9(1), 2.

Vaidya, A. (2008). Need for community-based primary prevention of coronary heart disease in Nepal. Kathmandu University Medical Journal, 6(4), 435-436.

Varghese, S. (2014). KNOWLEDGE REGARDING CARDIOVASCULAR RISK FACTORS AMONG PEOPLE IN SOUTH INDIA: A COMMUNITY BASED STUDY. Nitte University Journal of Health Sciences, 4(1), 42-45. 
ISSN: 2362-1303 (Paper) | elSSN: 2362-1311(Online)

WHO.(2010).HEALTHPROFILE:NEPAL.Retrivedfromhttp://www.worldlifeexpectancy.com/co untry-health-profile/nepal 